\title{
OUTPUT COMPLETENESS IN SEQUENTIAL MACHINES
}

\section{GENE F. ROSE}

In $\S 5$ of [1], Ginsburg considers states of complete sequential machines that yield all possible output words, calling them "output complete states. ${ }^{11}$ For any state that is not output complete, there is a shortest word not yielded by it, and Ginsburg points out that, among all machines in $n$ states, $r$ inputs and $m$ outputs, these shortest words have a least upper bound $k(m, n, r)$ depending only upon $m, n$ and $r$. The question of explicitly expressing $k(m, n, r)$ or an upper bound for it was left open. It follows as a special case of the present Theorem 2.4 that $2^{n-1}$ is an upper bound. Moreover, by Corollary 3.3 , for all sufficiently large $m$ and $r, 2^{n-1}$ is the least upper bound. An immediate implication of the primitive recursiveness of this bound is that the decision problem for output completeness is solvable.

The results for individual states were obtained as a special case of analogous findings for sets of states.

1. Definitions and notation. A (complete sequential) machine is a system consisting of a finite nonempty set of "states," a finite nonempty set of "inputs," a finite nonempty set of "outputs" and a rule that uniquely determines for each state $p$ and input $I$ a state $f(p, I)$ and an output $g(p, I)$. Finite nonempty sequences of inputs are represented by expressions called input words, defined thus: the input words of length 1 are the inputs; the input words of length $k+1 \geqq 2$ are the expressions of form $I X$ where $I$ is an input and $X$ is an input word of length $k$. Finite nonempty sequences of outputs are represented by output words, analogously defined.

The output sequence resulting from a given input sequence $X$ applied to an initial state $p$ is $g(p, X)$, where the range of the output function $g$ is extended by induction so that, if $I$ is an input and $X$ is an input word, $g(p, I X)=g(p, I) g(f(p, I), X)$.

For any nonempty set $P$ of states and any output word $Y$, " $P \vdash Y$ " (read " $P$ yields $Y$ ") means that $Y$ results from a suitable input sequence applied to some initial state in $P$; i.e. $P \vdash Y$ if and only if there is a state $p$ in $P$ and an input word $X$ such that $g(p, X)=Y$.

Received by the editors March 14, 1961.

${ }^{1}$ Cf. also [2]. Ginsburg's paper concerns quasi-machines. In this note, changes in terminology are made in order to paraphrase his remarks in the context of sequential machines. Thus Ginsburg's inputs and outputs become "input words" and "output words," and elements of input and output alphabets become simply "inputs" and "outputs." 
A set $P$ of states has degree 0 if and only if it is nonempty, degree $d(d>0)$ if and only if it yields all output words of length $d$. A set $P$ of states has proper degree $d$ if and only if it has degree $d$ and it does not have degree $d+1$.

A set $P$ of states is output complete if it yields all output words, otherwise $P$ is output incomplete.

For any output $E$ and state $p$, " $E(p)$ " denotes the set of states $q$ for which there exists an input $I$ such that $f(p, I)=q$ and $g(p, I)=E$. For any output $E$ and nonempty set $P=\left\{p_{1}, \cdots, p_{k}\right\}$ of states, " $E(P)$ " denotes the union of $E\left(p_{1}\right), \cdots, E\left(p_{k}\right)$.

2. Maximum proper degrees. The results of this section apply to an arbitrarily prescribed complete sequential machine with exactly $n$ distinct states.

2.1. LeMma. If $P$ is a set of states, $E$ an output and $Y$ an output word, then $P \vdash E$ if and only if $E(P)$ is nonempty, $P \vdash E Y$ if and only if $E(P) \vdash Y$.

Proof. The first part is obvious. For the second part, note that $P \vdash E Y$ if and only if there exist $p$ in $P$ and input word $I X$ for which $g(p, I X)=E Y$, hence if and only if there exist $p$ in $P$ and input $I$ for which $g(p, I)=E$ and $f(p, I) \vdash Y$. But if $p$ is in $P, g(p, I)=E$ and $f(p, I) \vdash Y$, then $f(p, I)$ is a member of $E(P)$ that $\vdash Y$; conversely, if $q$ is in $E(P)$ and $q \vdash Y$, then there exist $p$ in $P$ and input $I$ such that $g(p, I)=E, f(p, I)=q$ and $q \vdash Y$, and consequently $g(p, I)$ $=E$ and $f(p, I) \vdash Y$.

2.2. Lemma. $A$ set $P$ of states has degree $d(d \geqq 1)$ if and only if, for all outputs $E, E(P)$ has degree $d-1$.

Proof. There is an immediate consequence of 2.1.

2.3. Lemma. $A$ set $P$ of states has proper degree $d(d \geqq 1)$ if and only if, for all outputs $E, E(P)$ has degree $d-1$ and at least one $E(P)$ has proper degree $d-1$.

Proof. By 2.2, $P$ has proper degree $d$ if and only if all $E(P)$ 's have degree $d-1$ but not all have degree $d$.

2.4. Theorem. Any output incomplete set of $c$ distinct states has proper degree at most $2^{n}-2^{n-c}-1$. Hence in particular any output incomplete state has proper degree at most $2^{n-1}-1$.

Proof. Let $P$ be an output incomplete set of $c$ distinct states, with proper degree $d \geqq 1$. By 2.3 , there exists a sequence $P_{d-1}, \cdots, P_{0}$ of sets with respective proper degrees $d-1, \cdots, 0$. Because a set has 
the degree of any subset, none of the $P_{i}$ 's contains $P$; moreover, the $P_{i}$ 's, having distinct proper degrees, are distinct nonempty sets. Thus each $P_{i}$ is one of the $2^{n}$ subset $s$ of $n$ states; but of these subsets, $2^{n-c}$ contain $P$ and one is empty. Therefore $d$, the number of $P_{i}$ 's, is at most $2^{n}-2^{n-c}-1$.

2.5. Corollary. The decision problem for output completeness of sets of states, hence in particular of individual states, is solvable.

Proof. In view of 2.4 , a set $P$ of $c$ states of a given machine is output complete if and only if the finite set of all output words of length $2^{n}-2^{n-c}$ is obtained by applying the finitely many possible input words of the same length to initial states in $P$. It can be shown that there is an effective prescription to carry out this procedure for any given machine and set of states.

3. Construction of sets of states with specified proper degree. The results of this section apply to any given set $Q=\left\{q_{1}, \cdots, q_{n}\right\}$ of distinct states. It will be shown how, using only the given states, to construct a machine for which there is a set of states that has any prescribed proper degree consistent with 2.4 .

3.1. Lemma. Hy pothesis: $P_{i j}(i=0, \cdots, d ; j=1, \cdots, n)$ are arbitrary subsets of $Q$ such that, for each $j$, there is at least one nonempty $P_{i j}$. ConClusion: There is a complete sequential machine with states $Q$, outputs $E_{0}, \cdots, E_{d}$ and $n(d+1)$ inputs such that each $E_{i}\left(q_{j}\right)=P_{i j}$.

Proof. Let the inputs be $I_{01}, \cdots, I_{0 n} ; \cdots ; I_{d 1}, \cdots, I_{d n}$. Let $P_{i(1), 1}, \cdots, P_{i(n), n}$ be nonempty, as required by hypothesis. Define the state and output functions $f$ and $g$ as follows. If $P_{i j}$ is empty, $f\left(q_{j}, I_{i 1}\right)=\cdots=f\left(q_{j}, I_{i n}\right)=p_{i(j)}$, where $p_{i(j)}$ is an element of $P_{i(j), j}$, and $g\left(q_{j}, I_{i 1}\right)=\cdots=g\left(q_{j}, I_{i n}\right)=E_{i(j)}$; if $P_{i j}$ is not empty, list its elements, with repetitions as required, as $p_{1}, \cdots, p_{n}$ and let $f\left(q_{j}, I_{i 1}\right)$ $=p_{1}, \cdots, f\left(q_{j}, I_{i n}\right)=p_{n}, g\left(q_{j}, I_{i 1}\right)=\cdots=g\left(q_{j}, I_{i n}\right)=E_{i}$. It is a matter of routine to verify that the machine with states $Q$, the $I_{i j}$ 's as inputs, the $E_{i}$ 's as outputs, and state and output functions $f$ and $g$ as defined meets the requirements of the conclusion.

3.2. TheOREM. Hypothesis: $P_{0}, \cdots, P_{d}$ is a sequence of nonempty subsets of $Q$ such that, for all $i<j, P_{i}$ does not contain $P_{j}$. Conclusion: there is a complete sequential machine with states $Q$ such that the proper degree of each $P_{i}$ is $i$ and any subset of $Q$ included in no $P_{i}$ is output complete.

Proof. The case $d=0$ is obvious. For $d>0$, let the distinct outputs be $E_{0}, \cdots, E_{d}$ and define subsets $P_{i j}(i=0, \cdots, d ; j=1, \cdots, n)$ 
of $Q$ thus. For $j=1, \cdots, n, P_{0 j}=$ the empty set or $Q$ according as $q_{j}$ is in $P_{0}$ or not; for each $i$ from 1 to $d, P_{i j}=P_{i-1}$ or $Q$ according as $q_{j}$ is in $P_{i}$ or not. For each $j, P_{1 j}$ is nonempty. Therefore the $P_{i j}$ 's satisfy the hypothesis of 3.1 , so that there is a machine with states $Q$, outputs $E_{0}, \cdots, E_{d}$ and $n(d+1)$ inputs such that each $E_{i}\left(q_{j}\right)$ $=P_{i j}$. By construction, for any state $q_{j}$ in $Q, E_{0}\left(q_{j}\right)$ is the empty set or $Q$ according as $q_{j}$ is in $P_{0}$ or not and, for all $i$ from 1 to $d$, $E_{i}\left(q_{j}\right)$ is $P_{i-1}$ or $Q$ according as $q_{j}$ is in $P_{i}$ or not. An initial observation is that, for any set $P$ not contained in $P_{i}$, there is a $q_{j}$ in $P$ for which $E_{i}\left(q_{j}\right)=Q$, so that $E_{i}(P)=Q$. To prove the second part of the conclusion, let $P$ be contained in no $P_{i}$. If $P$ has degree $j$, then every $E_{i}(P)$ contains $P$ and therefore has degree $j$, so that, by $2.2, P$ has degree $j+1$; it follows by induction on $j$ that $P$ has every degree $j$, i.e. $P$ is output complete.

For the first part of the conclusion, we first prove by induction that for each $i, P_{i}, \cdots, P_{d}$ (hence in particular $P_{i}$ ) all have degree $i$. Basis. $P_{0}, \cdots, P_{d}$, being nonempty, have degree 0 . Induction step. Hypothesis: $P_{i}, \cdots, P_{d}$ have degree $i$. Then for all $j>i, E_{0}\left(P_{j}\right), \cdots$, $E_{j-1}\left(P_{j}\right)$ contain $P_{i}$ in view of the initial observation, and by construction $E_{j}\left(P_{j}\right), \cdots, E_{d}\left(P_{j}\right)$ contain $P_{j-1}, \cdots, P_{d-1}$ respectively. Conclusion (by 2.2 and the induction hypothesis): $P_{i+1}, \cdots, P_{d}$ have degree $i+1$. It now follows by induction on $i$ that $P_{i}$ has proper degree $i$. Thus $P_{0}$ has proper degree 0 , inasmuch as it is nonempty and no member of it yields $E_{0}$; if by induction hypothesis $P_{i}$ has proper degree $i$, then $E_{i+1}\left(P_{i+1}\right)=P_{i}$ also has proper degree $i$, so that by $2.3 P_{i+1}$ has proper degree $i+1$.

3.3. CoRollaRY. Given $1 \leqq c \leqq n$ and $d \leqq 2^{n}-2^{n-c}-1$, there is an $n$-state complete sequential machine with a c-state subset of proper degree $d$. In particular, if $d \leqq 2^{n-1}-1$, there is an $n$-state complete sequential machine with a state of proper degree $d$.

Proof. The corollary follows from the theorem by choosing an arbitrary $c$-state subset for $P_{d}$, listing, in order of increasing size, the $2^{n}-2^{n-c}-1$ nonempty sets not containing $P_{d}$, and taking $d$ of them for $P_{0}, \cdots, P_{d-1}$.

\section{REFERENCES}

1. S. Ginsburg, Some remarks on abstract machines, Trans. Amer. Math. Soc. 96 (1960), 400-444.

2. - An introduction to mathematical machine theory, Addison-Wesley, Reading, Mass., 1962.

System Development Corporation 